\title{
Durchführungsempfehlungen zur invasiven außerklinischen Beatmung
}

\author{
Gemeinsame Empfehlung der Deutschen Gesellschaft für Pneumologie (DGP), \\ der Deutschen Interdisziplinären Gesellschaft für Außerklinische Beatmung (DIGAB), \\ des Medizinischen Dienstes des Spitzenverbandes Bund der Krankenkassen e. V. (MDS) \\ und des AOK-Bundesverbandes (AOK-BV)
}

Recommendations for Invasive Home Mechanical Ventilation

Autoren

Institute
Koordinationskreis außerklinische Beatmung

W. J. Randerath ${ }^{1}$, N. Kamps ${ }^{2}$, J. Brambring ${ }^{3}$, F. Gerhard ${ }^{4}$, J. Lorenz ${ }^{5}$, F. Rudolf ${ }^{6}$, S. Rosseau ${ }^{7}$, A. Scheumann ${ }^{6}$, V. Vollmer ${ }^{8}$, W. Windisch ${ }^{9}$

Die Institutsangaben sind am Ende des Beitrags gelistet.

\section{Bibliografie}

DOI http://dx.doi.org/

10.1055/s-0030-1256121

Pneumologie 2011; 65:

72-88 @ Georg Thieme

Verlag KG Stuttgart · New York

ISSN 0934-8387

Korrespondenzadresse

Prof. Dr. med.

Winfried J. Randerath

Krankenhaus Bethanien

Institut für Pneumologie an der

Universität Witten/Herdecke

Klinik für Pneumologie und

Allergologie

Zentrum für Schlaf- und

Beatmungsmedizin

Aufderhöher Straße 169-175

42699 Solingen

Randerath@Klinik-bethanien.de

\section{Zusammenfassung \\ $\nabla$}

Aufgrund einer chronischen respiratorischen Insuffizienz ist bei einigen Patienten eine langfristige außerklinische Beatmung notwendig. Ihre Einleitung und Überwachung bedarf spezialisierter Kenntnisse der betreuenden Ärzte und Pflegekräfte, aber auch der zuständigen Mitarbeiter von Kostenträgern und des Medizinischen Dienstes der Krankenversicherung. Das vorliegende Papier stellt einen Konsens der beteiligten Fachgesellschaften, Kostenträger und deren medizinischen Beratungsdienst dar. Die Durchführungsempfehlungen basieren auf der S2-Leitlinie „Nichtinvasive und invasive Beatmung als Therapie der chronischen respiratorischen Insuffizienz“ [1]. Sie geben Empfehlungen zur Qualifikation von Ärzten und Pflegekräften in spezialisierten Zentren und in der außerklinischen Beatmung. Von wesentlicher Bedeutung ist das Überleitmanagement, das die medizinischen, technischen und organisatorischen Voraussetzungen für eine Entlassung aus der stationären Betreuung enthält. In der ambulanten Versorgung werden Aussagen zur notwendigen Hilfsmittelausstattung, zum Monitoring, zur Adjustierung der Beatmung und Frequenz erforderlicher Kontrolluntersuchungen getroffen. Die Empfehlungen werden ergänzt durch Anlagen, wie u.a. Überleitungsbögen, eine Checkliste zur Hilfsmittelbasisversorgung bei außerklinischer Beatmung sowie einen Entlassungsbrief-Entwurf.

\section{Vorwort}

$\nabla$

Im Jahr 2010 wurde die S2-Leitlinie „Nichtinvasive und invasive Beatmung als Therapie der chronischen respiratorischen Insuffizienz" der Deutschen Gesellschaft für Pneumologie und Beatmungsmedizin e.V. herausgegeben. Die Leitlinie beruht auf der Analyse der zunehmenden Zahl wissenschaftlicher Publikationen zum Thema und war aufgrund der rasant zunehmenden An-

\section{Abstract}

Due to chronic respiratory failure, a proportion of patients require long-term home ventilation therapy. The treating doctors, nurses and therapists, as well as employees of the health insurance provider, all require specialized knowledge in order to establish and monitor home ventilation. The following document represents a consensus formed by the participating specialist societies, the health insurers and their medical advisory services. The recommendations for accomplishing home mechanical ventilation are based on the "S2 Guidelines for Non-Invasive and Invasive Mechanical Ventilation for Treatment of Chronic Respiratory Failure“ [1], and provide advice about the necessary qualifications of medical and nursing practitioners working in specialised ventilation centres or in the home setting. Management of transfer, which comprises the medical, technical and organisational requirements for releasing the patient from hospital care, is of paramount importance. In outpatient care, the requirements for the recruitment of resources, monitoring of procedures, adjustment of ventilation, and frequency of check-ups are each addressed. The recommendations are supplemented by appendices which include patient transfer forms, checklists for the supply of basic resources for home ventilation, as well as a template for the letter of discharge from hospital.

wendung der außerklinischen Beatmung sowie der aktuellen gesundheitspolitischen Auseinandersetzung vor dem Hintergrund des Kostendruckes im Gesundheitswesen und der Notwendigkeit zur Gestaltung entsprechender Versorgungsstrukturen notwendig geworden. Sie ersetzt damit die bestehenden nationalen Empfehlungen. Um den Dialog zwischen den an der Betreuung außerklinisch beatmeter Patienten beteiligten Verbänden, insbesondere den medizinischen 
Fachgesellschaften Deutsche Gesellschaft für Pneumologie und Beatmungsmedizin (DGP), Deutsche interdisziplinäre Gesellschaft für außerklinische Beatmung (DIGAB) sowie Pflegediensten, dem Medizinischen Dienst der Krankenversicherung (MDK) und den Kostenträgern, zu verbessern, wurde der Koordinationskreis außerklinische Beatmung als ein gemeinsames Forum geschaffen.

Ziel ist es, klare Positionen für die außerklinische Beatmung aufzustellen und Durchführungshilfen für die praktische Anwendung zu geben, um die medizinische Versorgung beatmungspflichtiger Patienten in Deutschland nach einheitlichen Standards gewährleisten zu können.

Die hier vorliegenden Empfehlungen des Koordinationskreises außerklinische Beatmung sollen eine praktische Umsetzung der S2-Leitlinie „Invasive und nichtinvasive Beatmung als Therapie der chronischen respiratorischen Insuffizienz" darstellen, damit eine rasche und kontrollierte Entlassung maschinell beatmeter Patienten gewährleistet wird. Die Empfehlungen berücksichtigen schwerpunktmäßig Patienten, die nach invasiver Akutbeatmung auf der Intensivstation ein Weaningversagen erlitten haben. Hinsichtlich der Versorgung von Patienten, bei denen eine elektive Beatmungseinleitung erfolgen muss, ist die Erstellung einer gesonderten Empfehlung wünschenswert. Ziel des Koordinationskreises war es, Konsens in schwierigen Fragen der Versorgung herzustellen, um Fehlentwicklungen in der außerklinischen Betreuung entgegenzuwirken. Weiterführende Informationen finden sich auf den Seiten der Deutschen Interdisziplinären Gesellschaft für außerklinische Beatmung (DIGAB): www.digab.de.

Aus Gründen der besseren Lesbarkeit wird innerhalb der Empfehlungen in der Regel die männliche Schreibweise verwendet. Wir möchten deshalb darauf hinweisen, dass diese ausschließliche Verwendung der männlichen Form explizit als geschlechtsunabhängig verstanden werden soll.

\section{Einleitung}

In den letzten zwei Jahrzehnten haben Fortschritte in der Intensivmedizin und Beatmungstechnik die Prognose von Patienten mit einer respiratorischen Insuffizienz erheblich verbessert. Bei Patienten mit Weaningversagen ist jedoch auch nach Beendigung der Krankenhausbehandlung eine invasive außerklinische Beatmung medizinisch erforderlich, die in der Häuslichkeit des Patienten oder in Betreuungseinrichtungen, wie spezialisierten betreuten Wohngemeinschaften oder vollstationären Pflegeeinrichtungen, erfolgt [2].

Der Umgang mit einem Beatmungsgerät ist eine verantwortungsvolle Tätigkeit, die neben technischen Kenntnissen auch eine medizinische Ausbildung und praktische Erfahrung zur Voraussetzung hat. Eine Fehlfunktion der Atmung kann in kürzester Zeit zu Sauerstoffmangelzuständen mit lebenslangen Folgen für die Gehirnfunktion des Patienten und damit zu einer erheblichen Beeinträchtigung der Lebensqualität oder zum Tod des Patienten führen.

Die Einleitung und Überwachung einer invasiven Beatmung erfordert von den betreuenden Ärzten und Pflegekräften vertiefte Kenntnisse der Zusammenhänge von Atmungsregulation, Atemmechanik, Gasaustausch, Atempumpe und der gegenseitigen Beeinflussung der respiratorischen Funktion mit anderen Organfunktionen.

Eine Herausforderung stellt aber auch die Organisation und die Umsetzung der Versorgung im häuslichen Bereich dar, sodass auch eingebundene Mitarbeiter von Kostenträgern und des Medizinischen Dienstes der Krankenversicherung (MDK) über spezielle Kenntnisse verfügen sollten.

Ein frühzeitiges Einbinden der Kostenträger durch die entlassenden Einrichtungen ist für ein qualifiziertes Überleitmanagement unabdingbar.

Aufgrund der kontinuierlichen Zunahme der Patientenzahlen, der weiteren Differenzierung der Betreuungsstrukturen im außerklinischen Bereich und der kontinuierlichen technischen Weiterentwicklung ist eine Bewertung des aktuellen medizinisch-wissenschaftlichen Kenntnisstandes auf dem Gebiet der außerklinischen Beatmung erforderlich, um die Qualität in der Betreuung beatmeter Patienten zu sichern.

Dem Koordinationskreis außerklinische Beatmung (KKAB) ist dabei bewusst, dass derzeit Themen wie Finanzierung, Organisation und Durchführung der außerklinischen Beatmung in der Fachwelt intensiv diskutiert werden und die zukünftigen Entwicklungen noch nicht absehbar sind. Insofern berücksichtigen die folgenden Empfehlungen den aktuellen medizinisch-wissenschaftlichen Kenntnisstand sowie die derzeitigen Versorgungsstrukturen und bedürfen regelmäßiger Überarbeitung.

Krankenhäuser mit spezieller Expertise in der Beatmungsentwöhnung werden als Weaningzentren bezeichnet. Ein Weaningzentrum hat darüber hinaus weitreichende Erfahrungen im Bereich der außerklinischen Beatmung und der Überleitung von einem Versorgungsbereich in den andern.

Die Deutsche Interdisziplinäre Gesellschaft für außerklinische Beatmung (DIGAB) erarbeitet derzeit Kriterien für „Beatmungszentren“, die eine spezielle Expertise in der elektiven Einleitung außerklinischer Beatmung und der langfristigen Betreuung von außerklinisch beatmeten Patienten haben müssen.

\section{Krankenhausbehandlung}

Die Einleitung einer maschinellen Beatmung bei einem Patienten mit einer Atmungsinsuffizienz ist eine komplexe Maßnahme, die lebensbedrohliche Nebenwirkungen und Komplikationen haben kann, wie z.B. durch die fehlerhafte Einstellung oder Bedienung des Beatmungsgerätes oder des Beatmungszuganges.

Die Einleitung einer invasiven außerklinischen Beatmung nach Weaningversagen sollte immer unter stationären Krankenhausbedingungen unter Aufsicht von geschultem Personal erfolgen. Idealerweise wird die Einleitung in einem Weaningzentrum oder aber in Kooperation mit einem Weaningzentrum durchgeführt.

Ein Weaningzentrum ist ein Zentrum mit Expertise in der Indikationsstellung, dem Beginn und der Überwachung der außerklinischen Beatmung.

\subsection{Weaningzentrum}

In dem aktuell veröffentlichten vorläufigen Erhebungsbogen zur Akkreditierung von Weaningzentren (Quelle: www.pneumologie.de) muss ein Weaningzentrum über eine abgestufte Infrastruktur für die Behandlung maschinell beatmeter Patienten verfügen, die neben der Weaningeinheit auch eine Intensivstation und eine spezialisierte Heimbeatmungsstation umfasst. Ein Arzt (Qualifikation siehe 2.2) soll mehr als 10 Stunden am Tag ständig anwesend sein, über 24 Stunden muss in Notfallsituationen eine 
umgehende ärztliche Versorgung gewährleistet sein. Die räumlichen Bedingungen auf der Weaningstation müssen den Bedürfnissen wacher, langzeitbeatmeter Patienten (Ruhe, Tag-NachtRhythmus, ausreichend Raum, Lärmschutz) gerecht werden. Die personelle und technische Ausstattung sowie die Infrastruktur in der Klinik müssen so beschaffen sein, dass Versorgungslücken nicht entstehen. Eine nähere Beschreibung eines qualifizierten Weaningzentrums findet sich im Akkreditierungskatalog des „Weannet“ (www.pneumologie.de).

\subsection{Qualifikation von Ärzten und Pflegekräften im Weaningzentrum}

Die Beatmungstherapie ist eine originär ärztliche Aufgabe. Die Einstellung auf eine Beatmung darf nur durch einen in der Beatmung fachkundigen Arzt erfolgen.

Zur notwendigen Erfahrung des Arztes und zu den Anforderungen an seine Qualifikation sollten gehören:

1. Facharztqualifikation mit zusätzlich abgeschlossener Zusatzweiterbildung Intensivmedizin oder zumindest halbjähriger ganztägiger Erfahrung in der Intensivmedizin

2. eine mindestens 6-monatige Tätigkeit in einem Weaningzentrum oder auf einer Heimbeatmungsstation mit Ersteinstellung und Verlaufskontrolle von ausreichend ${ }^{1}$ Beatmungspatienten

3. die Durchführung von Schulungen von Pflegepersonen, Angehörigen und Patienten

4. Kenntnisse und Erfahrungen im Rahmen des Überleitmanagements

5. die Weiterbetreuung beatmeter Patienten ambulant und/oder stationär

Ärzte, die eine Neueinstellung auf ein Beatmungsgerät, eine Beatmungsgeräteumstellung oder eine Veränderung der Beatmungsparameter vornehmen, müssen umfassende Kenntnisse der Physiologie und Pathophysiologie der Atmung, der akuten und chronischen ventilatorischen Insuffizienz und ihren verschiedenen Ursachen, der Einstellungsparameter der Beatmungssysteme sowie detaillierte Kenntnisse der speziellen Beatmungssysteme aufweisen. Behandlungspflegerische Maßnahmen im Rahmen der Beatmung dürfen nur durch examinierte Pflegefachkräfte erbracht werden.

Examinierte Pflegefachkräfte oder Atmungstherapeuten dürfen bei Änderungen der Beatmungsgeräteeinstellung, des Zubehörs sowie des Beatmungszugangs nur auf ärztliche Anordnung hin tätig werden.

Die in die Betreuung eines beatmeten Patienten involvierten Ärzte und Pflegefachkräfte müssen über profunde Kenntnisse der Tracheostomaversorgung, der Methoden der Sekretelimination sowie über besondere Kenntnisse der im Rahmen der Beatmung eingesetzten Medizinprodukte, wie u.a. der Beatmungsgeräte, Trachealkanülen, Schlauchsysteme, Absaug- und Inhalationsgeräte sowie Befeuchtungssysteme, verfügen.

\footnotetext{
${ }^{1}$ Nach ausreichender Erfahrung mit dieser Regelung wird empfohlen, dass Mindestzahlen festgelegt werden.
}

\section{3 Überleitmanagement}

$\nabla$

Ist nach Ausschöpfung aller Maßnahmen nach Langzeitintensivtherapie keine vollständige Entwöhnung vom Respirator möglich, so besteht - unter bestimmten Voraussetzungen, siehe Pos. 3.1 - die Möglichkeit, die maschinelle Beatmung außerklinisch, in der Häuslichkeit oder einer spezialisierten Versorgungseinrichtung, fortzusetzen.

Nach den Kategorien der Budapester Konsensuskonferenz im Jahre 2005 [3] wird in Abhängigkeit vom individuellen Weaningprozess die Einteilung folgender Patientengruppen empfohlen:

- Gruppe 1 „Einfaches Weaning“: „Beim einfachen Entwöhnen vom Respirator sind die Patienten im 1. Versuch problemlos zu entwöhnen.

- Gruppe 2 „Schwieriges Weaning“: Eine „schwierige Entwöhnung vom Respirator" liegt vor, wenn ein Spontanatemversuch (Spontaneous Breathing Trial, SBT) erfolglos verläuft und bis zu 3 weitere SBT bzw. ein Zeitraum bis zu 7 Tage für das weitere Weaning benötigt wird.

- Gruppe 3 „Prolongiertes Weaning“: „Patienten, die mehr als 3 SBT oder mehr als 7 Tage zum erfolgreichen Weaning benötigen“.

Patienten der Gruppe 1 „Einfaches Weaning“ und Gruppe 2 „Schwieriges Weaning“ werden im Krankenhaus vollständig vom Beatmungsgerät entwöhnt. Die Patienten der Weaninggruppe 3 stellen ein sehr heterogenes Patientenkollektiv dar: während ein Teil der Patienten noch in der Klinik komplett vom Respirator entwöhnt werden kann, bleiben andere Patienten langfristig beatmungspflichtig. Dabei wird die Beatmung entweder als nichtinvasive oder als invasive Beatmung, in der Regel als außerklinische Langzeitbeatmung fortgeführt. Bei weiterhin invasiv beatmungspflichtigen Patienten der Weaninggruppe 3 sollte nur dann eine Überleitung und die Fortsetzung der Beatmung in der Häuslichkeit bzw. in spezialisierten Betreuungseinrichtungen angestrebt werden, wenn eine weitere Entwöhnung vom Beatmungsgerät im Rahmen der Krankenhausbehandlung nicht möglich und der Weaningprozess zunächst abgeschlossen ist.

Eine Entlassung mit dem Ziel, den Weaningprozess in der Häuslichkeit, in einer ambulanten oder stationären Betreuungseinrichtung fortzuführen, ist obsolet.

\subsection{Voraussetzungen für die Entlassung} aus der stationären Betreuung

Die Entlassung eines invasiv beatmeten Patienten in die Häuslichkeit bzw. in eine Versorgungseinrichtung kann erst dann veranlasst werden, wenn folgende medizinische, technische und organisatorische Voraussetzungen erfüllt sind:

Medizinische Voraussetzungen sind, dass

- die Umstellung von einer invasiven auf eine nichtinvasive Beatmung geprüft wurde,

- derzeit keine weiteren kurzfristigen Fortschritte (innerhalb der nächsten 4 Wochen) im Weaningprozess zu erwarten sind,

- alle Möglichkeiten der Beatmungsentwöhnung ausgeschöpft wurden und zumindest eine stabile Situation der Beatmung über $\geq 7$ Tage erreicht wurde,

- der Patient ohne kreislaufunterstützende Medikamente hämodynamisch stabil ist, 
- die Nierenfunktion des Patienten stabil ist oder bei dialysepflichtiger Niereninsuffizienz die Dialyseversorgung sicher gestellt ist und

- die Ernährung oral, enteral mittels Ernährungssonde oder parenteral sichergestellt ist.

Technische Voraussetzungen sind, dass

- hinsichtlich der medizinisch erforderlichen Hilfsmittel und Materialien eine Kostenübernahme von Seiten des Kostenträgers vorliegt,

- die medizinisch erforderlichen Hilfsmittel und Materialien am zukünftigen Aufenthaltsort vorhanden sind,

- Angehörige und soweit möglich der Patient selbst in der Anwendung/Bedienung der zum Einsatz kommenden Medizinprodukte geschult wurden.

Organisatorische Voraussetzungen sind, dass

- ein ärztlicher Entlassungsbrief (s. Anlage) mit den wesentlichen Angaben, u.a. zur Diagnose, Therapieempfehlung und Beatmungsgeräteeinstellung erstellt wurde,

- der Patient und die Angehörigen objektiv über die verschiedenen Möglichkeiten der Pflege und Betreuung durch z.B. den Sozialdienst des entlassenden Krankenhauses oder die Pflegeberatung (Pflegestützpunkte nach §7 SGB XI) informiert sind,

- Kontakt mit der weiter betreuenden Pflegeinstitution, dem Hausarzt/weiter betreuenden Vertragsarzt und Kostenträger aufgenommen und der entsprechende Überleitungsbogen für Hilfsmittel, ggf. Häusliche Krankenpflege gemäß §37 SGB V an den Kostenträger übermittelt wurde (s. Anlagen).

Es ist zu beachten, dass die Entlassung eines invasiv beatmeten Patienten mit Weaningversagen aus einem Krankenhaus, das selbst nicht Weaningzentrum ist, ebenfalls unter den genannten Kriterien erfolgen sollte. Das Krankenhaus sollte zudem ein Weaningzentrum konsultieren und den Überleitprozess in Kooperation mit dem Weaningzentrum durchführen.

Eine Entlassung mit dem Ziel, den Weaningprozess in der Häuslichkeit, in einer ambulanten oder stationären Betreuungseinrichtung fortzuführen, ist obsolet.

- Eine Überleitkonferenz, an der die weiter betreuende Pflegeinstitution, der Hausarzt/der weiter betreuende Vertragsarzt, der Kostenträger, ggf. vertraglich ermächtigte Leistungserbringer und der MDK, aber auch die Angehörigen/Eltern, Therapeuten des Patienten oder der Patient selbst teilnehmen, ist im Falle eines invasiv beatmeten Patienten dringend zu empfehlen.

- Vor Entlassung eines invasiv beatmeten Patienten in die Häuslichkeit oder in die Betreuungseinrichtung ist in jedem Fall ein Weaningzentrum zu konsultieren. Wenn kein Weaningpotenzial mehr vorhanden ist, muss der Patient nicht zwingend in ein Weaningzentrum verlegt werden.

Im Ergebnis der Überleitkonferenz werden die Resultate protokolliert und die entsprechenden Überleitungsbögen (siehe Anlagen) direkt an den Kostenträger weitergeleitet.

Der an den Kostenträger (die Krankenkasse) übermittelte Überleitungsbogen beinhaltet eine zusätzliche Stellungnahme des verantwortlichen Arztes des betreuenden Weaningzentrums.
Folgende Aussagen sollten enthalten sein:

- Ist im Falle des Patienten noch ein weiteres Weaning möglich?

- Ist die Verlegung des Patienten in ein Weaningzentrum erforderlich?

- Welches Weaningzentrum übernimmt den Patienten nach Überleitung in den außerklinischen Bereich zur langfristigen Betreuung?

Es wird empfohlen, sowohl in der entlassenden Klinik als auch beim Kostenträger ein sektorübergreifendes Fallmanagement zu etablieren. Diese Fallmanager stehen als Ansprechpartner sowie zur Kommunikation untereinander dem Patienten, den Angehörigen, den betreuenden Pflegekräften und dem behandelnden Vertragsarzt zur Verfügung. Sie koordinieren die Hilfsmittelversorgung gemäß §33 SGBV, die Feststellung der Pflegebedürftigkeit gemäß SGB XI (Pflegestufe) und ggf. die Beantragung häuslicher Krankenpflege gemäß §37 SGB V.

Die Kostenträger beauftragen im erforderlichen Umfang den Medizinischen Dienst der Krankenversicherung auf den gesetzlichen Grundlagen des SGB V und SGB XI zur Begutachtung. Hinsichtlich der zeitlichen Abfolge sei auf die $\bullet$ Abb. 1 u. 2 verwiesen.

\section{Ambulante Versorgung}

\section{1 Ärztliche außerklinische Versorgung}

Die Anforderungen an den betreuenden Arzt bei der Adjustierung eines Beatmungsgerätes nach bereits initiierter außerklinischer Beatmung sind die gleichen wie bei der Ersteinstellung auf die außerklinische Beatmung.

Zur notwendigen Erfahrung des Arztes und zu den Anforderungen an seine Qualifikationen gehören:

1. Facharztqualifikation mit zusätzlich abgeschlossener Zusatzweiterbildung Intensivmedizin oder zumindest halbjähriger ganztägiger Erfahrung in der Intensivmedizin

2. eine mindestens 6-monatige Tätigkeit in einem Weaningzentrum oder auf einer Heimbeatmungstation mit Ersteinstellung und Verlaufskontrolle von ausreichend ${ }^{2}$ Beatmungspatienten

3. die Durchführung von Schulungen von Pflegepersonen, Angehörigen und Patienten

4. Kenntnisse und Erfahrungen im Rahmen des Überleitmanagements

5. die Weiterbetreuung beatmeter Patienten ambulant und/oder stationär

Ärzte, die eine Veränderung von Beatmungsparametern vornehmen, müssen umfassende Kenntnisse der Physiologie und Pathophysiologie der Atmung, der akuten und chronischen ventilatorischen Insuffizienz und ihrer verschiedenen Ursachen, der Einstellungsparameter von Beatmungsgeräten sowie detaillierte Kenntnisse der speziellen Beatmungssysteme aufweisen. Sie müssen über profunde Kenntnisse der Tracheostomaversorgung, der Methoden der Sekretelimination sowie über besondere Kenntnisse der im Rahmen der Beatmung eingesetzten Medizinprodukte, wie u.a. der Beatmungsgeräte, Trachealkanülen, Schlauchsysteme, Inhalations- und Absauggeräte sowie Befeuchtungssysteme verfügen.

Da der den Patienten ambulant betreuende Hausarzt nicht regelhaft über Erfahrungen auf dem Gebiet der außerklinischen Beatmung verfügt, wird in der S2-Leitlinie „Nichtinvasive und invasive

\footnotetext{
${ }^{2}$ Nach ausreichender Erfahrung mit dieser Regelung wird empfohlen, dass
} Mindestzahlen festgelegt werden. 


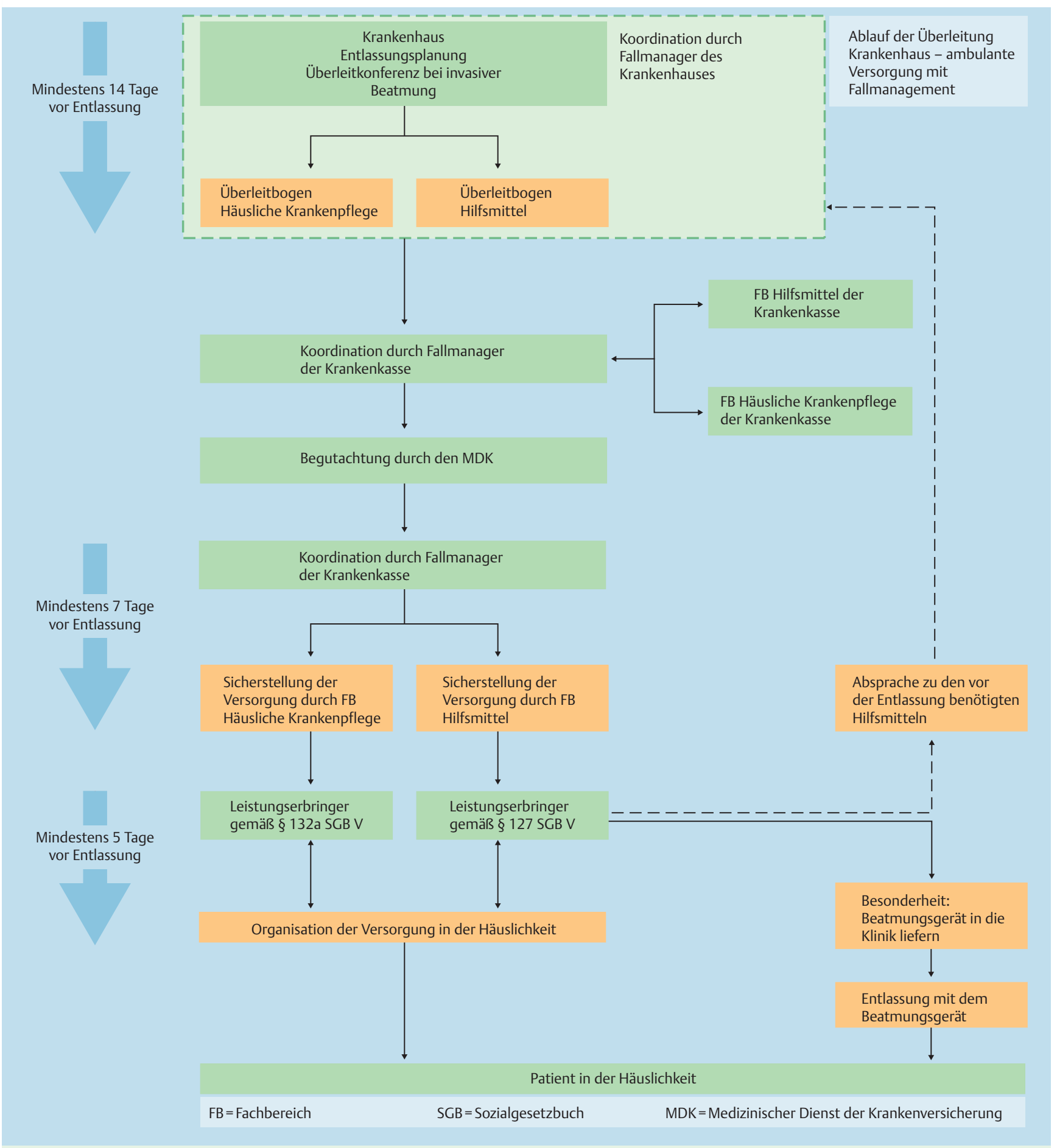

Abb. 1 Ablauf der Überleitung Krankenhaus - ambulante Versorgung mit Fallmanagement.

Beatmung als Therapie der chronischen respiratorischen Insuffizienz" empfohlen, dass ein in der außerklinischen Beatmung erfahrener Arzt hinzugezogen wird [1]. Hausbesuche sollten in der Betreuung außerklinisch invasiv beatmeter Patienten einen festen Bestandteil der Betreuung darstellen, da Praxisbesuche für Beatmete oft mit erheblichen Umständen verbunden sind.

Der betreuende Arzt ist für die gesamte Beatmung verantwortlich.

\subsection{Pflegerische Versorgung}

In der pflegerischen Versorgung beatmeter Patienten wird unterschieden zwischen der assistiven und fachpflegerischen Versorgung Beatmeter. In der assistiven Betreuung besteht die Möglichkeit der Versorgung im sogenannten Arbeitgebermodell, der Versorgung durch Pflegekräfte mit geringerem Qualifikationsniveau und der Versorgung durch Laienkräfte/Angehörige. Es sei hierbei auf die ausführlichen Ausführungen in der S2-Leitlinie „Nichtinvasive und invasive Beatmung als Therapie der chronischen respiratorischen Insuffizienz“ verwiesen. 


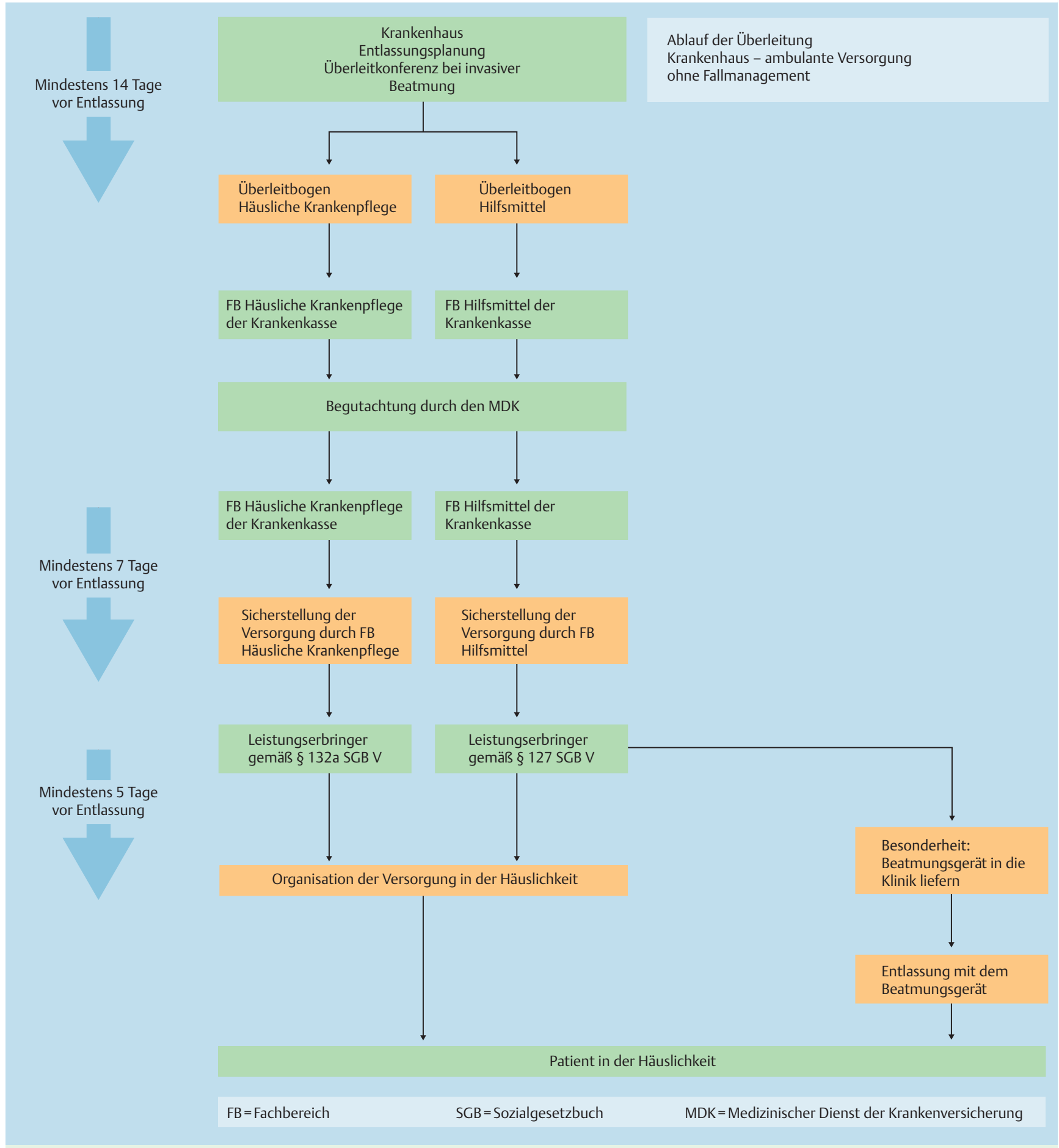

Abb. 2 Ablauf der Überleitung Krankenhaus - ambulante Versorgung ohne Fallmanagement.

\subsubsection{Häusliche Krankenpflege gemäß §37 SGB V}

Gemäß §37 Abs. 2 SGB V können „Versicherte... in ihrem Haushalt, ihrer Familie oder sonst an einem geeigneten Ort, insbesondere in betreuten Wohnformen, Schulen und Kindergärten, bei besonders hohem Pflegebedarf auch in Werkstätten für behinderte Menschen als häusliche Krankenpflege Behandlungspflege" erhalten, „wenn diese zur Sicherung des Ziels der ärztlichen Behandlung erforderlich ist.....

„Der Anspruch auf häusliche Krankenpflege besteht nur, soweit eine im Haushalt lebende Person den Kranken in dem erforderlichen Umfang nicht pflegen und versorgen kann."
Die in der vertragsärztlichen Versorgung verordnungsfähigen Maßnahmen der häuslichen Krankenpflege sind grundsätzlich dem der „Richtlinie des Gemeinsamen Bundesausschusses über die Verordnung von häuslicher Krankenpflege“ nach §92 Abs. 1 Satz $2 \mathrm{Nr} 6$ und Abs. 7 SGB V angefügten Leistungsverzeichnis zu entnehmen [5].

Entsprechend der Richtlinie sind dort nicht aufgeführte Maßnahmen „grundsätzlich nicht als häusliche Krankenpflege verordnungs- und genehmigungsfähig“.

„Nicht im Leistungsverzeichnis aufgeführte Maßnahmen der häuslichen Krankenpflege im Sinne von §37 SGB V sind in medizinisch 
zu begründenden Ausnahmefällen verordnungs- und genehmigungsfähig, wenn sie Bestandteil des ärztlichen Behandlungsplans sind, im Einzelfall erforderlich und wirtschaftlich sind und von geeigneten Pflegekräften erbracht werden sollen."

Gemäß aktueller Richtlinie zur Verordnung häuslicher Krankenpflege gemäß §37 SGBV von 06/2010 ist eine Anpassung und Überprüfung der Einstellungen des Beatmungsgerätes an Vitalparameter (z.B. Atemgase, Herzfrequenz, Blutdruck) auf Anordnung des Arztes bei beatmungspflichtigen Erkrankungen (z.B. hohe Querschnittslähmung, Zustand nach Schädel-Hirntrauma); Überprüfung der Funktionen des Beatmungsgerätes, ggf. Austausch bestimmter Teile des Gerätes (z. B. Beatmungsschläuche, Kaskaden, 02-Zellen) verordnungsfähig.

Nicht als häusliche Krankenpflege verordnungsfähig sind Maßnahmen der ärztlichen Diagnostik und Therapie. Insofern sind ein außerklinisches Weaning und behandlungspflegerische Maßnahmen mit der Zielsetzung eines Weanings nicht verordnungsfähig.

Die Dauer der personellen Überwachung wird für den einzelnen Patienten durch den für die Beatmung verantwortlichen Vertragsarzt festgelegt.

\subsubsection{Rückzugspflege}

Der pflegerische Versorgungsumfang in der außerklinischen Beatmung sollte sich immer dem Bedarf des Patienten individuell anpassen. Durch Anleitung und Schulung der Angehörigen und des Patienten selbst können diese im Rahmen ihrer Möglichkeiten nach und nach eigenständig einen Teil der Pflege übernehmen. Somit ist ein anteilsmäßiger Rückzug des Pflegedienstes den täglichen Stundenversorgungsumfang betreffend möglich. Dies ist auch für eine größtmögliche Autonomie der Familie erstrebenswert.

So wie sich der Allgemeinzustand des Patienten verbessern kann, ist auch eine Verschlechterung möglich. In dem Fall muss der pflegerische Versorgungsumfang des Patienten ggf. wieder erhöht werden.

\subsubsection{Qualifikation der betreuenden Pflegekräfte}

In der Häuslichkeit oder spezialisierten Betreuungseinrichtungen sind die Pflegekräfte häufig über längere Zeit alleine beim Patienten tätig. Ein spezialisierter Arzt ist nicht, wie im Weaningzentrum, ständig anwesend, sondern muss in besonderen Situationen hinzugerufen werden.

Daraus resultiert, dass die fachlichen Ansprüche an den - beatmete Patienten betreuenden - ambulanten Pflegedienst ebenso hoch sind wie an die beatmete Patienten betreuenden Pflegekräfte im Weaningzentrum.

Alle Pflegekräfte, die selbstständig am hochgradig respiratorabhängigen Patienten (i.d. R. tägliche Beatmungsdauer >18 Stunden) tätig sind, müssen über die o.g. Kenntnisse (Punkt 2.2) verfügen. Zusätzlich ist eine Ausbildung als examinierte/r Krankenschwester/-pfleger, Gesundheitspfleger/-in, Kinderkrankenschwester/-pfleger oder Altenpfleger/-in (mit dreijähriger Ausbildung) notwendig.

Mitarbeitende von Pflegediensten mit geringerer Qualifikation (Krankenpflegehelfer/-in, Arzthelfer/-in) dürfen nicht eigenverantwortlich in der Betreuung der Beatmung tätig werden.
- Die Pflegeperson darf nur Maßnahmen übernehmen, für die sie qualifiziert ist.

- Der betreuende Arzt hat sich zu überzeugen, dass die Pflegeperson, die mit der Durchführung einer ärztlicherseits angeordneten Maßnahme beauftragt wird, diese beherrscht.

Hinsichtlich der Qualifikation der betreuenden Pflegekräfte wird auf die in der S2-Leitlinie „Nichtinvasive und invasive Beatmung als Therapie der chronischen respiratorischen Insuffizienz" im Kapitel 6.4 ausführlich ausgeführte Qualifikation des außerklinischen Pflegeteams verwiesen.

Für den Beatmungsbereich muss eine speziell qualifizierte examinierte Fachkraft als Fachbereichsleitung vorgehalten werden. Diese übernimmt intern die fachliche Aufsicht und Verantwortung für die außerklinische Versorgung und die Qualifikation der am Patienten eingesetzten Pflegekräfte.

Die Fachbereichsleitung dient innerhalb der Pflegedienste als Wissensmultiplikator. Die Fachbereichsleitung muss nicht zwingend die Pflegedienstleitung in einer Person sein. Jedem beatmeten Patienten und seinen Angehörigen wird die ihm zugeordnete Fachbereichsleitung bekannt gemacht und eine weitere Kommunikation bei Bedarf ermöglicht.

Zusätzlich zu einer 3-jährigen Ausbildung als examinierter Gesundheits- und Krankenpfleger, Gesundheits- und Kinderkrankenpfleger ist eine der folgenden Qualifikationen für die Fachbereichsleitung erforderlich:

- Atmungstherapeut mit pflegerischer Ausbildung

- Fachgesundheits- und Krankenpflege für Anästhesie- und Intensivpflege

- mindestens drei Jahre Berufserfahrung im Beatmungsbereich (Intensivstation, Intermediate-Care-Station oder außerklinische Beatmung) in den letzten fünf Jahren und Fortbildung (mindestens 200-Stunden-Kurs der medizinischen Fachgesellschaften bzw. pflegerischen Berufsverbände, anerkanntes strukturiertes berufsbegleitendes Kursprogramm in der Beatmungspflege). Diese Kurse müssen von der DIGAB anerkannt sein und werden auf der Homepage der DIGAB aufgeführt.

Alle Pflegekräfte, die selbstständig und eigenverantwortlich am Beatmungsgerät abhängiger Patienten (fachpflegerische Versorgung) tätig sind, müssen zusätzlich zu einer 3-jährigen Ausbildung als examinierte Gesundheits- und Krankenpfleger, Gesundheits- und Kinderkrankenpfleger oder Altenpfleger eine der folgenden Qualifikationen aufweisen:

- Atmungstherapeut

- Fachgesundheits- und Krankenpflege für Anästhesie- und Intensivpflege

- mindestens ein Jahr Berufserfahrung im Beatmungsbereich (Intensivstation, Intermediate Care-Station oder außerklinische Beatmung) innerhalb der letzten 5 Jahre. Abweichend kann die Zusatzqualifikation auch durch einen strukturierten Kurs zur Beatmungspflege erworben werden. Diese Kurse müssen entsprechend strukturiert und von der DIGAB anerkannt sein. Sie werden auf der Homepage der DIGAB aufgeführt.

\subsubsection{Außerklinische Versorgung von Patienten mit Langzeitbeatmung}

Die außerklinische Versorgung von Patienten mit Langzeitbeatmung ist in der Häuslichkeit des Patienten unter bestimmten Umständen möglich. Dies setzt allerdings ein geeignetes soziales 
Umfeld und möglichst auch Barrierefreiheit voraus. Ist eine Weiterversorgung des außerklinisch beatmeten Patienten im eigenen Haushalt ausgeschlossen, so besteht die Möglichkeit die außerklinische Beatmung in spezialisierten Betreuungseinrichtungen, wie

- vollstationären Pflegeeinrichtungen und

b betreuten Wohngemeinschaften, fortzuführen.

\subsubsection{Unterscheidung zwischen assistiver und} fachpflegerischer Versorgung

Bei der assistiven Versorgung (niedriges Qualifikationsniveau) wird in der Regel eine Hilfestellung ohne examinierte Qualifikation benötigt, während die fachpflegerische Versorgung (hohes Qualifikationsniveau) immer auf eine qualifizierte, durch examiniertes Pflegepersonal durchgeführte Behandlung angewiesen ist.

Die Entscheidung, ob es sich um eine Versorgung auf hohem oder niedrigem Niveau handelt, obliegt dem „erst“-verordnenden Krankenhausarzt und geschieht in Absprache mit dem Patienten. Die außerklinische Weiterversorgungsform hängt primär vom Wunsch des Beatmeten und den familiären Ressourcen ab. Der Patient hat Anspruch auf eine unabhängige, umfassende Beratung, verbunden mit dem Hinweis auf entsprechende Selbsthilfeorganisationen, da Gespräche mit gleichfalls Betroffenen sehr hilfreich für die Akzeptanz und die Gestaltung des Alltags sein können.

\subsubsection{Assistive Versorgung Beatmeter}

Es gibt verschiedene Möglichkeiten der assistiven Versorgung außerklinisch beatmeter Menschen:

- Versorgung im sog. „Arbeitgebermodell“

Die Patienten übernehmen selbstständig und eigenverantwortlich die Auswahl ihrer persönlichen Assistenten sowie die Verantwortung für deren Befähigung.

- Versorgung durch Pflegekräfte mit geringem Qualifikationsniveau

Derzeit können Mitarbeiter geringer Qualifikation (z. B. Krankenpflegehelfer/innen) nur eingeschränkt (z. B. in der Grundpflege) in der Versorgung beatmeter Patienten (Beatmungsstation, Wohngemeinschaften) tätig werden.

- Laienkräfte - Angehörige

Laienkräfte, in der Regel aus dem Kreis der Familie oder dem sozialen Umfeld, übernehmen die pflegerische Versorgung mit Einverständnis des Patienten.

Eine Anleitung von Laienkräften durch den Pflegedienst wird empfohlen. Die in die Pflege involvierten Laienkräfte (z. B. Angehörige) sollen dabei in der Durchführung der grund- und behandlungspflegerischen Maßnahmen angeleitet bzw. unterstützt und im Hinblick auf das Beherrschen der Maßnahmen kontrolliert werden, um diese Tätigkeiten dauerhaft selbst durchführen oder dauerhaft Hilfestellung bei der eigenständigen Durchführung der Maßnahmen geben zu können. Die Verordnung kann auf Basis der Richtlinie des Gemeinsamen Bundesausschusses über die Verordnung von häuslicher Krankenpflege (Häusliche Krankenpflege-Richtlinie) [5] erfolgen.

Die Hinzunahme externer Experten, die bzgl. des Krankheitsbildes und der therapeutischen Maßnahmen erfahren sind, wird zumindest zur Einarbeitung bei den assistiven Versorgungsmodellen empfohlen.

\subsubsection{Besonderheiten in der außerklinischen Versorgung von Kindern mit Langzeitbeatmung}

Die Betreuung von Kindern mit invasiver außerklinischer Beatmung und insbesondere von Kleinkindern erfordert eine besondere, zusätzlich den pädiatrischen Fachbereich betreffende, Expertise. Idealerweise werden die Kinder in der Klinik und außerklinisch von einem multidisziplinären Team betreut.

Bei Kindern ist eine ventilatorische Insuffizienz in der Regel Folge eines komplexen, oft progredienten Krankheitsbildes. Diagnostik, Beurteilung der Prognose, Indikationsstellung, Einleitung einer Beatmung und die langfristige medizinische und pflegerische Betreuung von beatmeten, meist mehrfach behinderten Kindern erfordern besondere Kenntnisse und Fertigkeiten aller an der Betreuung des Kindes beteiligten Berufs- und Personengruppen. Da außerklinisch beatmete pädiatrische Patienten sich in vielerlei Hinsicht von Erwachsenen unterscheiden, sollte ihre Behandlung in einem multidisziplinären Team aus Kinderärzten, Kinderkrankenpflegepersonal, Physiotherapeuten und anderen Berufsgruppen mit entsprechender Erfahrung und Zusatzqualifikation erfolgen.

Insbesondere beatmete Säuglinge und Kleinkinder benötigen eine hohe Aufmerksamkeit, engmaschige Kontrolle und sicheres Monitoring.

Sofern die Versorgung eines vom Beatmungsgerät abhängigen Kindes durch eine Betreuungseinrichtung erfolgt, müssen die Fachbereichsleitung und die Versorgung vorrangig durch Gesundheits- und Kinderkrankenpfleger (nachrangig durch Gesundheits- und Krankenpfleger) mit den in Kapitel 4.2.3 genannten optionalen Zusatzqualifikationen für die pädiatrische Beatmungspflege gewährleistet sein.

Eine sichere und erfolgreiche außerklinische Beatmung muss das familiäre und häusliche Umfeld des Patienten berücksichtigen und durch medizinische, pflegerische und psychosoziale Unterstützungsangebote begleitet werden. Die Bildung von pädiatrischen Kompetenzzentren, die eine außerklinische Beatmung einleiten, die Behandlung des Kindes koordinieren und mit Kinderund Jugendärzten, ambulanten Pflegediensten, Betreuungseinrichtungen, Sozialpädiatrischen Zentren (SPZ) und heimatnahen Kliniken zusammenarbeiten, kann die Versorgung von langzeitbeatmeten Kindern verbessern.

\subsubsection{Kontrolluntersuchungen}

Zu den ambulant möglichen Nachsorgeuntersuchungen gehören die Überprüfung der Therapietreue (Compliance) und die Überprüfung der voreingestellten Parameter am Beatmungsgerät sowie die Überprüfung der Trachealkanüle und des Zubehörs.

Eine Kontrolle unter Erfassung wesentlicher Kenngrößen der Atmung, insbesondere der Sauerstoffsättigung und des Kohlendioxid-Partialdruckes sollte erstmalig nach 3, und dann alle 6 bis 12 Monate in einer spezialisierten Klinik (z.B. einem Weaningzentrum) erfolgen. Im Einzelfall können die Intervalle verkürzt oder verlängert werden.

Im Rahmen der Kontrolluntersuchungen sollte auch im Langzeitverlauf regelmäßig eine Einschätzung des Weaningpotenzials des beatmeten Patienten erfolgen, ferner sind die Versorgungs- und Therapieziele zu prüfen.

\subsection{Hilfsmittelversorgung}

Die Richtlinie des Gemeinsamen Bundesausschusses über die Verordnung von Hilfsmitteln in der vertragsärztlichen Versorgung (Hilfsmittel-Richtlinie/HilfsM-RL, aktuelle Fassung siehe www.g-ba.de [4]) ist für die an der vertragsärztlichen Versor- 
gung teilnehmenden Ärzte und ärztlich geleiteten Einrichtungen, die Krankenkassen sowie Leistungserbringer verbindlich. Sie dient einer nach den Regeln der ärztlichen Kunst und unter Berücksichtigung des allgemein anerkannten Standes der medizinischen Erkenntnisse ausreichenden, zweckmäßigen und wirtschaftlichen Versorgung der Versicherten mit Hilfsmitteln.

Sobald die Entlassung eines beatmeten Patienten - unter Beachtung der in Punkt 4.1 aufgeführten Voraussetzungen - geplant wird und der Ort der weiteren Betreuung (in der Häuslichkeit oder einer Betreuungseinrichtung) feststeht, sollte die verantwortliche Krankenkasse als Kostenträger involviert werden, um die Bereitstellung der benötigten Hilfsmittel und Materialien zu gewährleisten.

Unabdingbar für eine zeitgerechte Bereitstellung der erforderlichen Hilfsmittel und Materialien ist eine frühzeitige Information (spätestens 14 Tage vor Entlassung) direkt an den Kostenträger - Fallmanager - mit Übermittlung der entsprechenden ärztlichen Verordnungen.

Hinsichtlich der Hilfsmittel-Basisversorgung invasiv beatmeter Patienten wird auf die Anlage 4 dieser Empfehlungen verwiesen. Auf der Grundlage der vorliegenden Informationen (Einstellung des in der Klinik zur Verfügung stehenden Beatmungsgerätes) prüft die Krankenkasse zunächst, ob die Möglichkeit eines Wiedereinsatzes eines geeigneten Beatmungsgerätes und ggf. auch weiterer Hilfsmittel besteht.

Das Beatmungsgerät muss bereits in der Klinik (also vor der Entlassung des Patienten) zur Verfügung stehen, die Einstellung des Beatmungsmodus und der Beatmungsparameter obliegt dem verantwortlichen Arzt.

Es muss eine Einweisung in alle für die Versorgung notwendigen Medizinprodukte erfolgen. Alle in die Pflege involvierten Personen müssen durch den Geräteprovider eingewiesen werden. Die Einweisung muss spätestens am Tag der Entlassung durchgeführt werden.

Vor endgültiger Entlassung des Patienten in die außerklinische Versorgung muss die Finanzierung der Versorgung gesichert sein.

\subsubsection{Monitoring/personelle Überwachung}

Der für die Beatmung des Patienten verantwortliche Arzt legt unter Berücksichtigung der individuellen Situation des Patienten neben dem Umfang des technischen Monitorings auch die Dauer der personellen Überwachung des Patienten - nach Rücksprache mit den Angehörigen und dem Patienten - fest.

Weder ein Pulsoxymeter noch ein Kapnometer gehören im Falle eines beatmeten Erwachsenen zur Hilfsmittel-Basisversorgung. Über die Notwendigkeit ist im Einzelfall zu entscheiden. Es sei ergänzend auf die weitergehenden Ausführungen der S2-Leitlinie "Nichtinvasive und invasive Beatmung als Therapie der chronischen respiratorischen Insuffizienz" verwiesen.

\subsection{2 Überwachung der Beatmungsgeräteeinstellung/}

Adjustierung

Bei einer kontinuierlichen oder intermittierenden Beatmung sind jeweils zu Schichtbeginn die aktuellen Beatmungsparameter am Beatmungsgerät zu ermitteln und zu dokumentieren.

Das Beatmungsgerät inklusive Schlauchsystem und Zubehör sowie der Beatmungszugang sind jeweils zu Schichtbeginn auf einen regelrechten Aufbau und Funktionalität zu überprüfen.

Ebenso muss das Ersatzbeatmungsgerät einmal täglich auf Funktion und Parametereinstellung überprüft werden.

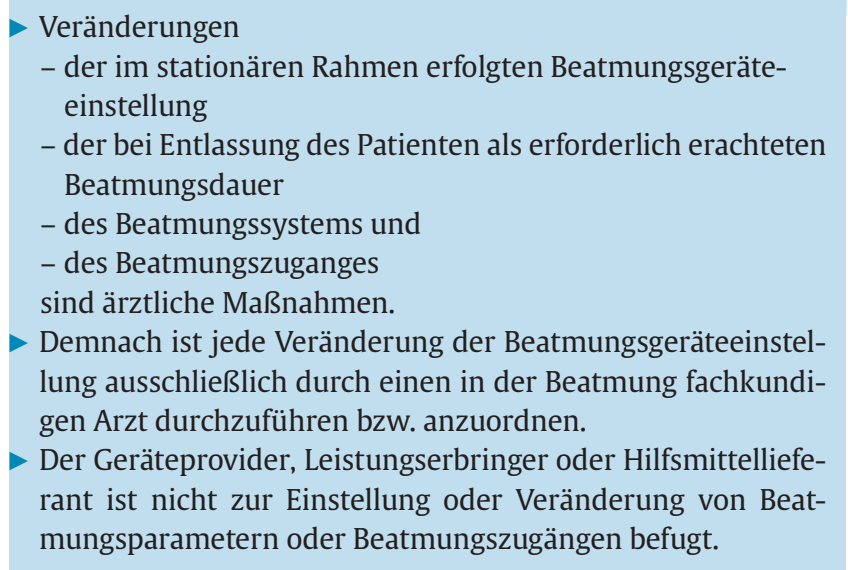

Die Umstellung von Beatmungsparametern durch den Arzt oder auf Anweisung eines Arztes erfordert anschließend eine mehrstündige Überwachung des Patienten unter Erfassung wesentlicher Kenngrößen der Atmung, insbesondere der Sauerstoffsättigung und des Kohlendioxid-Partialdruckes im arteriellen/kapillaren Blut oder der Ausatemluft auch im Schlaf. Die persönliche Überwachung durch spezialisiertes Personal ist notwendig. Ein beatmungserfahrener Arzt muss jederzeit unmittelbar verfügbar sein.

Eine klinische Verschlechterung des Zustandes des Patienten unter der außerklinischen Beatmung bedarf zunächst immer der ärztlichen Diagnostik und ergebnisabhängig der spezifischen Therapie.

Durch den verantwortlichen Arzt des betreuenden Weaningzentrums kann für den Fall akuter Verschlechterung eine individuelle Bedarfsanordnung für den jeweiligen Patienten festgelegt werden. Dies eröffnet dem ambulant weiter betreuenden Arzt bzw. Pflegedienst in der Notfallsituation auf der Basis der klinischen Symptomatik und ggf. Blutgasanalysen Handlungsoptionen. Die Bedarfsanordnung ersetzt allerdings nicht das Hinzuziehen eines in der Beatmung fachkundigen Arztes bzw. eine umgehende Vorstellung des Patienten bei dem die Beatmung betreuenden Arzt zur weiteren Diagnostik bzw. Therapie (siehe Kapitel 4.4)

\subsubsection{Aufgaben des Leistungserbringers}

Leistungserbringer haben nach $§ 127$ SGBV Verträge mit den Krankenkassen abgeschlossen und gelten somit als Vertragspartner der Krankenkassen. Sie sind berechtigt, im Auftrage der Krankenkassen auf Basis der bestehenden individuellen Verträge die Versicherten mit Hilfsmitteln zu beliefern. Diese Leistungserbringer werden umgangssprachlich auch als Hilfsmittellieferant oder Provider bzw. Geräteprovider bezeichnet. Die Gewährleistung der technischen Betreuung ist rund um die Uhr notwendig. 
Der Geräteprovider, Leistungserbringer oder Hilfsmittellieferant ist nicht zur Einstellung oder Veränderung von Beatmungsparametern oder Beatmungszugängen befugt.

\subsubsection{Austausch von Beatmungsgeräten und Interfaces} Beatmungsgeräte unterscheiden sich zum Teil erheblich in ihrem Triggerverhalten, der Priorität zum Erreichen bestimmter Zielparameter und in ihrer Druck- und Volumenapplikation. Daher kann eine Umstellung eines Beatmungsgerätes anderen Typs oder Herstellers, aber auch eines Beatmungszuganges (Umstellung invasive Beatmung auf nicht invasive Beatmung) nur unter subtiler Austestung am Patienten unter Überwachung durch einen beatmungserfahrenen Arzt im Rahmen eines stationären Krankenhausaufenthaltes, z.B. im betreuenden Weaningzentrum, erfolgen.

Eine Umstellung von einem Beatmungsgerät auf ein Beatmungsgerät anderen Typs oder Herstellers durch direkte Übertragung der voreingestellten Beatmungsparameter kann in der außerklinischen Beatmung ohne Kontrolle der Beatmungsqualität wegen der unterschiedlichen Geräteeigenschaften für den Patienten gefährlich sein und muss deswegen in der Häuslichkeit oder Betreuungseinrichtung unterbleiben.

Ein direkter Austausch baugleicher Beatmungsgeräte (gleicher Typ, gleiche Software, gleiche Artikelnummer) unter Beibehaltung aller Parameter oder des gleichen Beatmungszuganges ist durch den fachkundigen Arzt oder auf ärztliche Anordnung durch die qualifizierte Pflegefachkraft möglich.

\subsection{Indikationen zur Wiederaufnahme in das Krankenhaus}

Eine klinische Verschlechterung des Zustandes des Patienten im Rahmen der außerklinischen Beatmung ist als erneute Erkrankung zu werten und bedarf einer ärztlichen Therapie. Eine stationäre Neueinstellung der Beatmung oder eine Intensivierung der Behandlung sind auch dann erforderlich, wenn

- sich die Dauer der Spontanatmung erheblich verkürzt,

- sich die Kapazität der Spontanatmung erheblich verbessert,

- sich die Sekretproduktion anhaltend vermehrt,

- komplizierende Erkrankungen eintreten (z.B. Pneumonie, Exazerbation einer COPD, dekompensierte Herz- oder Niereninsuffizienz)

- sich der Gesamtzustand anhaltend verschlechtert.

Eine weitere Indikation zur stationären Aufnahme ist i.d.R. bei Umstellung des Beatmungsgerätes oder des Beatmungszugangs gegeben (siehe 4.3.4).

\section{Sterbebegleitung}

Eine außerklinische Beatmung hat die anhaltende Stabilität und das Überleben des Patienten bei angemessener Lebensqualität zum Ziel.

Die Fortführung oder Beendigung lebenserhaltender Maßnahmen, also auch der Beatmung, hängen vom Willen des Patienten ab. Dazu sind Patientenverfügungen und Vorsorgevollmachten hilfreich. Kann der Patient seinen Willen nicht äußern, ist vom Arzt der mutmaßliche Wille zu ermitteln, dabei sind Angehörige und betreuende Pflegekräfte einzubeziehen. Die Grundsätze der Bundesärztekammer sind zu berücksichtigen, die Etablierung eines ethischen Konsils ist zu empfehlen.
Bei der Sterbebegleitung muss in jedem Fall eine umfassende pflegerische Versorgung des Patienten gewährleistet sein. Die Beherrschung von Atemnot, Übelkeit, Schmerzen und anderen Krankheitsbeschwerden, der Erhalt der bestmöglichen Lebensqualität sowie die Behandlung psychologischer, sozialer und spiritueller Probleme besitzen in der Palliativversorgung höchste Priorität. Insofern kann auch hier eine permanente Interventionsbereitschaft - jedoch aus palliativer Indikation - erforderlich sein.

In enger Zusammenarbeit mit Patient, Angehörigen, Palliativmedizinern, Pflegenden, Sozialarbeitern sowie Geistlichen wird ein therapeutisches Konzept erarbeitet, das die Linderung des Leidens in den Vordergrund stellt.

\section{Übersicht über die Anlagen}

$\nabla$

Anlage 1: Deckblatt zu den Überleitungsbögen Hilfsmittel und Behandlungspflege

Anlage 2: Überleitungsbogen Hilfsmittel für invasiv beatmete Patienten

Anlage 3: Überleitungsbogen Behandlungspflege für invasiv beatmete Patienten

Anlage 4: Basisversorgung - Hilfsmittel

Anlage 5: Vorläufiger Verlegungsbericht

Anlage 6: Teilnahme- sowie Datenschutzerklärung und

Erklärung zur Entbindung von der Schweigepflicht

\section{Links}

$\nabla$

- Für die Zertifizierung von Weaningzentren: www.pneumologie.de

- Für die Beatmungstherapie: www.digab.de

- „Richtlinie des Gemeinsamen Bundesausschusses über die Verordnung von Hilfsmitteln in der vertragsärztlichen Versorgung (Hilfsmittel-Richtlinie/HilfsM-RL) in der Neufassung vom 16. Oktober 2008, veröffentlicht im Bundesanzeiger 2009, Nr. 61 S. 462, in Kraft getreten am 7. Februar 2009“: http://www.g-ba.de/downloads/62-492-309/RL-HilfsmittelNeufassung-2008-10-16.pdf

- „Richtlinie des Gemeinsamen Bundesausschusses über die Verordnung von häuslicher Krankenpflege (Häusliche Krankenpflege-Richtlinie) in der Neufassung vom 17. September 2009, veröffentlicht im Bundesanzeiger, Beilage vom 9. Februar 2010, zuletzt geändert am 15. April 2010, veröffentlicht im Bundesanzeiger S. 2214, in Kraft getreten am 26. Juni 2010“: http://www.g-ba.de/downloads/62-492-436/RL-HKP-201004-15.pdf

\section{Interessenkonflikt}

$\nabla$

Die Autoren geben an, dass kein Interessenkonflikt besteht. 


\section{Institute}

${ }^{1}$ Krankenhaus Bethanien, Institut für Pneumologie an der Universität Witten/Herdecke, Klinik für Pneumologie und Allergologie, Zentrum für Schlaf- und Beatmungsmedizin, im Auftrag der Deutschen Gesellschaft für Pneumologie und Beatmungsmedizin (DGP) und der Deutschen Interdisziplinären Gesellschaft für Außerklinische Beatmung (DIGAB)

2 Medizinischer Dienst des Spitzenverbandes Bund der Krankenkassen e.V. (MDS), Essen, im Auftrag des AOK-Bundesverbandes

3 Heimbeatmungsservice Brambring Jaschke $\mathrm{GmbH}$, Unterhaching, im Auftrag der Deutschen Interdisziplinären Gesellschaft für Außerklinische Beatmung (DIGAB)

4 isb Ambulante Dienste gGmbH, Wuppertal, im Auftrag der Deutschen Interdisziplinären Gesellschaft für Außerklinische Beatmung (DIGAB)

5 Klinikum Lüdenscheid, Klinik für Pneumologie, Infektiologie, Schlafmedizin und Internistische Intensivmedizin, Lüdenscheid, im Auftrag der Deutschen Gesellschaft für Pneumologie und Beatmungsmedizin (DGP)

6 AOK-Bundesverband, Berlin

7 Charité-Universitätsmedizin Berlin, CC12 Medizinische Klinik m.S., Infektiologie und Pneumologie, Charité Zentrum für außerklinische Beatmung und Sauerstofftherapie (CABS), Campus Mitte, Berlin, im Auftrag der Deutschen Gesellschaft für Pneumologie und Beatmungsmedizin (DGP) und der Deutschen Interdisziplinären Gesellschaft für Außerklinische Beatmung (DIGAB)

8 MDK Berlin-Brandenburg e.V., Berlin, im Auftrag des AOK-Bundesverbandes

${ }_{9}$ Universitätsklinikum Freiburg, Abteilung Pneumologie, Freiburg, im Auftrag der Deutschen Interdisziplinären Gesellschaft für Außerklinische Beatmung (DIGAB)

\section{Literatur}

1 Windisch WB, Budweiser S, Dellweg D et al. S2-Leitlinie. Nichtinvasive und invasive Beatmung als Therapie der chronischen respiratorischen Insuffizienz, herausgegeben von der Deutschen Gesellschaft für Pneumologie und Beatmungsmedizin e.V. Pneumologie 2010; 64: 207 240

2 Randerath W, Lorenz J, Windisch $W$ et al. Betreuung von Patienten mit maschineller Beatmung unter häuslichen und heimpflegerischen Bedingungen. Pneumologie 2008; 62: 305 - 308

3 Schönhofer $B$. Weaning vom Respirator beginnt mit der Intubation. Pneumologe 2008; 5: $150-162$

4 Richtlinie des Gemeinsamen Bundesausschusses über die Verordnung von Hilfsmitteln in der vertragsärztlichen Versorgung (HilfsmittelRichtlinie/HilfsM-RL) in der Neufassung vom 16. Oktober 2008 viB, Nr. 61 S. 462, in Kraft getreten am 7. Februar 2009

5 Richtlinie des Gemeinsamen Bundesausschusses über die Verordnung von häuslicher Krankenpflege (Häusliche Krankenpflege-Richtlinie) in der Neufassung vom 17. September 2009 viB, Beilage vom 9. Februar 2010, zuletzt geändert am 15. April 2010, veröffentlicht im Bundesanzeiger S. 2214, in Kraft getreten am 26. Juni 2010 


\section{Anlage 1}

Stammdaten - Deckblatt für Überleitungsbogen - Hilfsmittel/Behandlungsplege - für invasiv beatmete Patienten

Empfehlung des Koordinationskreises außerklinische Beatmung

Leistungsträger/Kostenträge

Anschrift

Telefon/Fax:

Patient/in: Name, Vorname

Geburtsdatum

Krankenversicherungs-Nr.

Anschrift

Angehörige/r: Name, Vorname

Anschrift

Telefon/Fax:

Gerichtlich bestellter Betreuer/in: Name, Vorname

Anschrift

Telefon/Fax:

Entlassende Klinik (Name)

Anschrift/Station

Telefon/Fax:

Ambulant betreuender Vertragsarzt

Name, Vorname

Anschrift

Telefon/Fax

Betreuendes Weaningzentrum

Anschrift

Kontaktaufnahme am:

Mitbetreuender Arzt:

Telefon/Fax:

Vollstationäre Betreuungseinrichtung (Name)

Anschrift

Telefon / Fax:

Ambulanter Pflegedienst (Name)

Anschrift

Telefon/Fax

Entlassungs-Diagnose

- ICD 10

- Klartext

- Ggf. weitere Diagnosen

Weaningpotenzial

\section{Beatmungsgerät}

Beatmungsgeräteeinstellung

Beatmungsdauer innerhalb von 24 Std.:

$\square$ nein $\square$ ja:

$\square$ Betreuung in der Häuslichkeit $\quad \square$ Betreuende Wohneinrichtung

$\square$ nein $\quad \square$ ja

festgestellt am:

durch:

Empfehlung zur Kontrolluntersuchung am:

Artikelbezeichnung:

Beatmungsform:

$\square$ druckgesteuert $\quad \square$ volumengesteuert

$\square$ assistiert

$\square$ kontrolliert

Beatmungsmodus:

Beatmungsparameter:

IPAP: mbar $_{\text {EPAP }}$ (PEEP) :__ mbar

Atemfrequenz
Volumensicherung notwendig $\square$ ja $\quad \square$ nein

$\square$ tagsüber von _ bis__ Std.

$\square$ nachts von_ bis__ Std.

Anzahl der maschinell nicht unterstützten Spontanatmungsphasen:

Maximaldauer: von __ bis___ $/ 24$ Std. 


\section{Anlage 2}

Überleitungsbogen - Hilfsmittel - für invasiv beatmete Patienten

Empfehlung des Koordinationskreises außerklinische Beatmung

Deckblatt siehe Anlage 1

\begin{tabular}{l|lll} 
Bewusstseinslage & Ansprechbar: $\quad \square$ nein & $\square$ ja & \\
& Orientiert: & $\square$ nein & $\square$ ja, zu $\square$ Ort
\end{tabular} Zeit $\square$ Person

Kommunikation

Besteht die grundsätzliche Befähigung, sich lautsprachlich zu äußern:

$\square$ nein $\quad \square$ ja, Muttersprache:

Kommunikationsmittel:

Mobilität

\begin{tabular}{|c|c|c|c|}
\hline Mobilisiert: & $\square$ nein & $\square$ ja, $\square$ bis zur Bettkante & $\square$ in den Rollstuhl \\
\hline Stehfähig: & $\square$ nein & $\square$ ja, $\square$ selbständig & $\square$ mit Unterstützung \\
\hline Gehfähig: & $\square$ nein & $\square$ ja, $\square$ selbständig & $\square$ mit Unterstützung \\
\hline
\end{tabular}

Tracheostoma

$\square$ seit:

$\square$ dilatativ angelegtes Tracheostoma

$\square$ chirurgisch angelegtes Tracheostoma

Trachealkanüle

$\square$ einteilig (ohne Innenkanüle) $\square$ zweiteilig (mit Innenkanüle)

$\square$ mit Manschette (Cuff) $\quad \square$ ohne Manschette (Cuff)

$\square$ gefenstert/gesiebt

$\square$ Nutzung eines Sprechventils in der beatmungsfreien Zeit

Artikelbezeichnung:

Artikelnummer:

Kanülenwechsel

erfolgt durch:

$\square$ Arzt $\quad \square$ Pflegefachpersonal $\quad \square$ Angehörige $\quad \square$ Patient

Sauerstoffinsufflation

$\square$ nein

$\square \mathrm{ja}, \square$ während der Beatmung $\quad \square$ im beatmungsfreien Intervall

Dauer der Sauerstoffinsufflation innerhalb von 24 Std.: ___ Std.

Sauerstoff-Flow in Ruhe von:___ $1 / \mathrm{min}$

Sauerstoff-Flow unter Belastung von:___ $1 / \mathrm{min}$

Sauerstoff-Flow unter Beatmung von:___ $1 / \mathrm{min}$

Sauerstoff-Flow im beatmungsfreien Intervall von:

Tägliche Außer-Haus-Mobilität: bis zu Stunden

Sauerstofftherapiegerät

$\square$ stationäres Sauerstofftherapiegerät

$\square$ mobiles Sauerstofftherapiegerät

mit Demand-System $\quad \square$ ja $\quad \square$ nein

$\square$ Sauerstoff-Notfalleinheit (10 I Druckgasflasche)

Blutgasanalyse

Datum:

Ohne $\mathrm{O}_{2}$ (wenn möglich)

\begin{tabular}{|l|l}
\hline ohne Beatmung & mit Beatmung
\end{tabular} $1 / \min$

\begin{tabular}{|r|}
\hline \\
\hline $\mathrm{PaO}_{2}$ \\
\hline $\mathrm{PaCO}_{2}$ \\
\hline $\mathrm{pH}$ \\
\hline
\end{tabular}

Atemgasklimatisierung

Passive Atemgasbefeuchtung

„künstliche Nasen“

$\square$ nein

$\square$ ja, $\quad \square$ passiv $\square$ aktiv

$\square$ während der invasiven Beatmung

Artikelbezeichnung:

Artikelnummer:

$\square$ im beatmungsfreien Intervall

Artikelbezeichnung:

Artikelnummer:

Absauggeräte

$\square$ Netzabhängiges Absauggerät Artikelbezeichnung:

$\square$ Netzunabhängiges Absauggerät

Hustenassistent

Inhalationsgerät

Arzt-Stempel, IK

\section{Artikelbezeichnung:}

$\square$ nein

$\square$ ja

$\square$ nein $\quad \square$ ja
$\quad$ Artikelbezeichnung:

Inhalation

$\square$ im beatmungsfreien Intervall

$\square$ obere Atemwege

Partikelgröße im Durchmesser:

$\square<3 \mu \mathrm{m} \quad \square 3 \mu \mathrm{m}$ bis $6 \mu \mathrm{m}$

$\square$ während der Beatmung

$\square$ untere Atemwege

$\square 6 \mu \mathrm{m}$ bis $12 \mu \mathrm{m}$ 
Anlage 3

Überleitungsbogen - Behandlungspflege - für invasiv beatmete Patienten

Empfehlung des Koordinationskreises außerklinische Beatmung

\section{Deckblatt siehe Anlage 1}

Patient/in: Name, Vorname

Geburtsdatum

Krankenversicherungs-Nr.

\begin{tabular}{l|ll} 
Pflegestufe nach SGB XI & $\square$ nein & $\begin{array}{l}\square \text { beantragt } \\
\text { Pflegestufe: }\end{array}$ \\
\hline Betreuungseinrichtung & $\square$ I $\square$ II $\square$ III \\
\hline vollstationär $\quad \square$ ambulant
\end{tabular}

$\square$ Assistive Versorgung im $\square$ Arbeitgeber-Modell „persönliches Budget“

$\square$ durch Pflegekräfte mit geringerem Qualifikationsniveau

$\square$ Fachpflegerische Versorgung

$$
\square \text { Laienkräfte/Angehörige }
$$

Ist Häusliche Krankenpflege gemäß § 37 SGB V erforderlich? $\square$ ja $\square$ nein

Bitte entsprechende Verordnung analog Muster 12 beifügen.

Gründe für die Anwesenheit einer geeigneten Pflegeperson zur Interventionsbereitschaft:

Wie viele Stunden (innerhalb von 24 Stunden) ist die Anwesenheit mit Interventionsbereitschaft

einer in der Beatmungspflege versierten Pflegeperson erforderlich? Stunden

- Wird gewährleistet durch: über

Stunden/Tag

- Wird gewährleistet durch: über Stunden/Tag 


\section{Anlage 4}

Hilfsmittel-Basisversorgung für invasiv beatmete Patienten Empfehlung des Koordinationskreises außerklinische Beatmung

\begin{tabular}{|c|c|}
\hline Artikel / Hilfsmittel & Invasive Beatmung \\
\hline $\begin{array}{l}\text { Beatmungsgerät (mit Zulassung für } \\
\text { die invasive außerklinische Beatmung) }\end{array}$ & $\begin{array}{l}\text { - Bei einer Beatmungsdauer von mehr als } 16 \text { Stunden ist in der Regel das Erfordernis eines zweiten } \\
\text { baugleichen Beatmungsgerätes gegeben. } \\
\text { - Ein zweites Beatmungsgerät ist erforderlich, wenn der Patient im Falle eines Gerätedefekts nicht über eine } \\
\text { ausreichende Spontanatmung verfügt, um ärztliche Hilfe (i.d.R. die nächste geeignete Klinik mit einer } \\
\text { Beatmungsmöglichkeit) zu erreichen. Hierbei muss die Wechselhaftigkeit des Krankheitsbildes (verlängerte } \\
\text { Beatmungszeiten bei Infekten!), ggf. Lage der Wohnung/Betreuungseinrichtung beachtet werden. }\end{array}$ \\
\hline Beatmungsschlauch/„Gänsegurgel““ & $X^{(1)}$ \\
\hline Externer Akku für das Beatmungsgerät & Im medizinisch begründeten Einzelfall \\
\hline Filter (Lufteinlass- und Auslassfilter) & $\begin{array}{l}\qquad X^{(1)} \\
\text { Hinsichtlich der zusätzlichen Versorgung mit Luftauslassfiltern (Bakterienfiltern) bei Nutzung von HMEF wird } \\
\text { entsprechend MPG auf die Herstellerfachinformation verwiesen. }\end{array}$ \\
\hline Inhalationsgerät & Im medizinisch begründeten Einzelfall \\
\hline Atemgasklimatisierung & $\begin{array}{l}\text { - Passive Befeuchtung: } \\
\text { - Basisversorgung Erwachsene } 1 \text { HME/Tag } \\
\text { - Wechselintervall gemäß MPG entsprechend der Herstellerfachinformation } \\
\text { - Kontraindikationen für den Einsatz von HME gemäß Herstellerfachinformation beachten, } \\
\text { z.B. bei Hypersekretion } \\
\text { - Aktive Befeuchtung } \\
\text { - Im medizinisch begründeten Einzelfall }\end{array}$ \\
\hline Absauggerät & Zwei Absauggeräte: ein netzabhängiges und ein zweites netzunabhängiges Absauggerät \\
\hline $\begin{array}{l}\text { Absaug-, Verbindungsschlauch, } \\
\text { Bakterienfilter, Fingertip, } \\
\text { Absaugkatheter }\end{array}$ & $X^{(1)}$ \\
\hline Trachealkanüle & 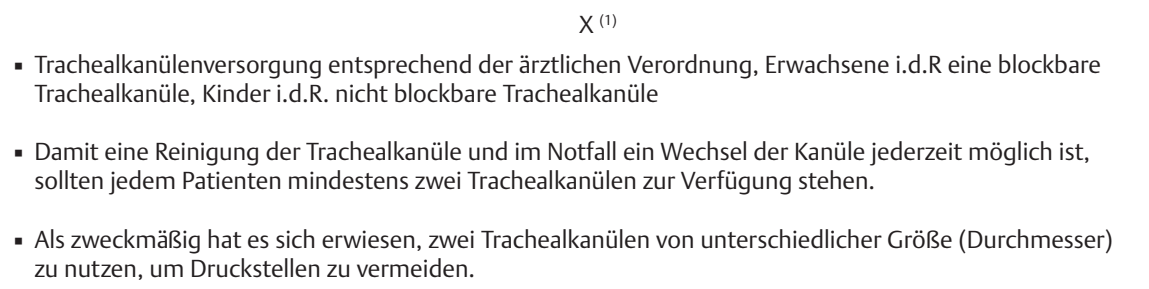 \\
\hline Pulsoxymeter & Im medizinisch begründeten Einzelfall \\
\hline Beatmungsbeutel, Testlunge & $X^{(1)}$ \\
\hline
\end{tabular}

(1) Wechselintervall gemäß MPG entsprechend der Herstellerfachinformation 


\title{
Anlage 5
}

\section{Entlassende Klinik \\ $X X X X X X X X X X X X$ \\ $X X X X X X X X X X X X$}

Weiterbetreuender Vertragsarzt

Datum, $X X X X X X X$

\section{Vorläufiger Verlegungsbericht}

\author{
Sehr geehrter Dr. XXXXXXXX,
}

wir berichten über unseren gemeinsamen Patienten Herrn / Frau XXXXXX, der / die sich vom XXXXX bis XXXX in unserer stationären Behandlung aufgrund einer $X X X X X X X X X X X X$ befand. $X X X X X X X X X X X X X X$

Ein weiteres Weaning d. Pat. war aufgrund XXXXXXXXXXXX nicht möglich.

Weitere Angaben siehe Anlage.

Aktuelle Beatmungseinstellung (Heimbeatmungsgerät XXXX, VO am XXXX)

Beatmungsmodus:

Beatmungsparameter:

Beatmungsdauer:

Aktuelle Medikation siehe Anlage

Letzter Trachealkanülenwechsel am:

Empfohlene Kontrolluntersuchung am:

\section{Anlage 6}

Überleitmanagement für außerklinisch Beatmete:

Schriftliche Erklärung des Versicherten bzw. seines gesetzlichen Vertreters gemäß § 11 Abs. 4 SGB V in Verbindung mit § 284 Abs. 1 Nr. 13 SGB V

Hinweis für den Versicherten: Als Versicherte/r haben Sie einen Anspruch auf ein qualifiziertes Überleitmanagement. Dieses Überleitmanagement soll insbesondere dazu dienen, Probleme beim Übergang zwischen den verschiedenen Versorgungsbereichen zu vermeiden. Damit die betroffenen Leistungserbringer Ihnen insbesondere auch eine sachgerechte Anschlussversorgung zukommen lassen können, ist es unerlässlich, dass diese (also die beteiligten Leistungserbringer) die erforderlichen Informationen zu Ihrer Person und Ihrer Erkrankung erhalten.
Ihre Krankenkasse nimmt hier eine aktive Rolle ein und unterstützt die Beteiligten, damit Ihre medizinische Versorgung reibungslos erfolgen kann. Der Gesetzgeber hat im Sozialgesetzbuch festgelegt, dass die im Zusammenhang mit dem Überleitmanagement erforderliche Übermittlung von Daten nur mit Einwilligung und nach vorheriger Information des Versicherten (oder seines gesetzlichen Vertreters) erfolgen darf. Die nachstehende Erklärung dient dazu, diesem gesetzlichen Erfordernis nachzukommen:
Ich bin damit einverstanden, dass für die Durchführung eines qualifizierten Überleitmanagements nach § 11 Abs. 4 SGB V in Verbindung mit § 284 Abs. 1 Nr. 13 SGB V für außerklinisch Beatmete meine Krankenkasse (Name der Krankenkasse) die mit meiner Erkrankung zusammenhängenden Behandlungsdaten zur Verfügung gestellt bekommt. Insoweit entbinde ich meine behandelnden Ärzte bzw. Leistungserbringer von der Schweigepflicht.

Ich bin ferner damit einverstanden, dass die für dieses Überleitmanagement erforderlichen - und bei meiner Krankenkasse vorliegenden - Informationen zur Person, zur Erkrankung und deren Behandlung (beispielsweise: Krankenhausentlassungs- und Befundberichte, Angaben aus dem Gutachten zur Feststellung der Schwerpflegebedürftigkeit, Informationen über bislang bezogene Hilfsmittel-, Heilmittel-, Pflege- und Krankentransportleistungen) von meiner Krankenkasse den betroffenen Leistungserbringern und ggf. dem Medi- zinischen Dienst der Krankenversicherung im erforderlichen Umfang zur Verfügung gestellt bzw. übermittelt werden. Zu den betroffenen Leistungserbringern zählen insbesondere der weiterbehandelnde Arzt, die gewählte Betreuungseinrichtung bzw. der gewählte Pflegedienst, der Heil- bzw. Hilfsmittelleistungserbringer und ggf. der Rehabilitations- bzw. Kostenträger. Die Datenübermittlung dient insbesondere dazu, die weitere Behandlung und Pflege zu organisieren und die notwendigen Leistungen zu koordinieren.

Mir ist bekannt, dass ich dieses Einverständnis jederzeit widerrufen kann. Eine entsprechende formlose (telefonische oder schriftliche) Information an meine Krankenkasse (Name der Krankenkasse) ist dazu ausreichend. Im Falle meines Widerrufs werden alle Behandlungsdaten - nach Ablauf der gegebenenfalls bestehenden gesetzlichen Aufbewahrungsfristen - gelöscht.

$\overline{\text { Ort }} \overline{\text { Datum }} \quad \overline{\text { Unterschrift }}$

